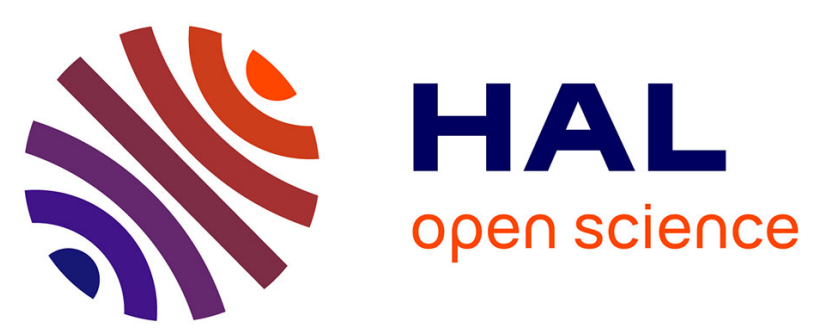

\title{
A system model for ultrasonic NDT based on the Physical Theory of Diffraction (PTD)
}

M. Darmon, V. Dorval, A. Kamta Djakou, L. Fradkin, S. Chatillon

\section{To cite this version:}

M. Darmon, V. Dorval, A. Kamta Djakou, L. Fradkin, S. Chatillon. A system model for ultrasonic NDT based on the Physical Theory of Diffraction (PTD). Ultrasonics, 2016, 64, pp.115-127. 10.1016/j.ultras.2015.08.006 . cea-01845392

\section{HAL Id: cea-01845392 https://hal-cea.archives-ouvertes.fr/cea-01845392}

Submitted on 7 Jan 2019

HAL is a multi-disciplinary open access archive for the deposit and dissemination of scientific research documents, whether they are published or not. The documents may come from teaching and research institutions in France or abroad, or from public or private research centers.
L'archive ouverte pluridisciplinaire HAL, est destinée au dépôt et à la diffusion de documents scientifiques de niveau recherche, publiés ou non, émanant des établissements d'enseignement et de recherche français ou étrangers, des laboratoires publics ou privés. 


\title{
A system model for ultrasonic NDT based on the Physical Theory of Diffraction (PTD)
}

\author{
M. Darmon ${ }^{\mathrm{a}, *}$, V. Dorval $^{\mathrm{a}}$, A. Kamta Djakou ${ }^{\mathrm{a}, \mathrm{b}}$, L. Fradkin ${ }^{\mathrm{c}}$, S. Chatillon ${ }^{\mathrm{a}}$ \\ ${ }^{\text {a } C E A, ~ L I S T, ~ D e p a r t m e n t ~ o f ~ I m a g i n g ~ \& ~ S i m u l a t i o n ~ f o r ~ N o n d e s t r u c t i v e ~ T e s t i n g, ~ F-91191 ~ G i f-s u r-Y v e t t e, ~ F r a n c e ~}$ \\ ${ }^{\mathrm{b}}$ Laboratoire d'Acoustique de l'Université du Maine (LAUM), UMR CNRS 6613, 72085 Le Mans, France \\ ${ }^{\mathrm{c}}$ Sound Mathematics Ltd., Cambridge CB4 2AS, UK
}

\begin{abstract}
Simulation of ultrasonic Non Destructive Testing (NDT) is helpful for evaluating performances of inspection techniques and requires the modelling of waves scattered by defects. Two classical flaw scattering models have been previously usually employed and evaluated to deal with inspection of planar defects, the Kirchhoff approximation (KA) for simulating reflection and the Geometrical Theory of Diffraction (GTD) for simulating diffraction. Combining them so as to retain advantages of both, the Physical Theory of Diffraction (PTD) initially developed in electromagnetism has been recently extended to elastodynamics. In this paper a PTD-based system model is proposed for simulating the ultrasonic response of crack-like defects. It is also extended to provide good description of regions surrounding critical rays where the shear diffracted waves and head waves interfere. Both numerical and experimental validation of the PTD model is carried out in various practical NDT configurations, such as pulse echo and Time of Flight Diffraction (TOFD), involving both crack tip and corner echoes. Numerical validation involves comparison of this model with KA and GTD as well as the Finite-Element Method (FEM).
\end{abstract}

\section{Introduction}

Classical ultrasonic inspection methods, pulse echo, tandem and Time of Flight Diffraction (TOFD) allow one to detect cracks by interpreting their specular or diffraction echoes. In Non Destructive Testing modelling nowadays plays an important role in assessing detection capability and conceiving inspections. System models have been developed to predict results of ultrasonic inspection in a range of applications $[1,2]$. These models simulate the propagated beam as well as its interaction with the flaws and reception by the probe of the waves scattered by the flaws.

Two classical semi-analytical scattering models are used in the literature to simulate interaction with cracks: the Kirchhoff approximation (KA) [3] and Geometrical Theory of Diffraction (GTD) [4]. They are based on different approximations and have complementary regions of validity. KA relies on an integral over the flaw surface of the product of the Geometrico-Elastodynamic (GE) field and the free space Green's function. It is used to deal with reflections from planar-like cracks (maybe, containing several planar facets) as well as volumetric voids, such as spherical or hemispherical holes and Side Drilled Holes (SDH) [5]. The corresponding

\footnotetext{
* Corresponding author. Tel.: +33 169082288.

E-mail address: michel.darmon@cea.fr (M. Darmon).
}

inspection model requires the meshing of defect surface. KA is particularly suitable for simulating direct reflection from flaws as well as corner effects [1,3]. KA can also deal with anisotropy [6] and impedance (non-rigid) interfaces [7]. GTD is suitable for simulating scattering by crack edges, away from specular angles and forward paths. The corresponding model requires the meshing of the flaw edge. Unlike GTD, KA does not model edge diffraction correctly and unlike KA, GTD is not suitable for describing reflections. Moreover, the GTD coefficients diverge near incident and reflection shadow boundaries.

Chapman [3] has presented the first complete system model, which could use either KA or GTD to simulate the ultrasonic response of a planar crack in an isotropic material but it had been limited to 2D configurations, in which the crack is perpendicular to the incident plane. KA models have been then extended to 3D [1,5]. Several GTD-based system models [8,9] have been developed for 2D configurations, particularly TOFD, in which cracks are detected using their edge diffracted echoes. The system model [10] is the only GTD-based model to deal with 3D CAD-defects and 3D inspection configurations.

Choosing between models based on KA or GTD requires expertise. Therefore, development of a generic model that can account for both reflection and edge diffraction is of great interest in NDT applications. Recently an original 2D approach based on the 
Huygens-Fresnel diffraction theory has been proposed [11] for evaluating the edge diffracted signal in TOFD configuration. The model uses both the monochromatic far field expressions derived by Miller and Pursey [12] and reciprocity principle. The amplitude of diffracted wave behind a vertical crack is obtained by summing up contributions of virtual elementary sources (situated on a virtual flaw), which are located inside the specimen section outside the crack. Since the Miller and Pursey's solution describes the GE Green's function of the 2D Lamb problem (the linear source located on the free surface of a semi-infinite elastic medium), the proposed method is equivalent (as shown in [13]) to applying KA over the virtual flaw. Due to this equivalence, the Huygens-Fresnel model is likely to lead to significant prediction errors in diffracted shear waves (see Section 3.2). To account for reflection, the HuygensFresnel model [11] has to be modified to sum up virtual sources on the real scatterer as done in [11] for computing the component backwall echo. This method [11] also seems to be able to deal with the incident shadow boundary, as illustrated by simulation results presented in Fig. 6 in [11]. This figure cannot correspond to the TOFD configuration for a planar component described in Fig. 5 of [11], since in this configuration the incident wave cannot reach the receiver and the incident shadow boundary cannot be located at the observation angle of $45^{\circ}$ as Fig. 6 suggests. The method [11] is also restricted by being applicable only in TOFD and 2D configurations and since it relies on the Miller and Pursey's solution, it cannot describe scattered head waves or regions surrounding critical rays $[14,15]$.

In this paper an ultrasonic system model is proposed, accounting for both reflection and diffraction, for 3D crack-like flaws in 3D configurations. It is based on the Physical Theory of Diffraction (PTD). PTD is a scattering model which was initially developed in electromagnetism [16] and which overcomes the limitations of both KA and GTD so that the resulting scattered field is spatially uniform. PTD has recently been extended to elastodynamics in [17]. Elastodynamic PTD is incorporated here in the generic ultrasonic system model of the CIVA software package [18]. This article describes this PTD-based system model and shows its prediction capabilities for 3D crack-like defects. Additionally, the region of the PTD model's validity is extended in this paper to cover transition zones surrounding critical rays where the shear diffracted waves and head waves interfere.

The paper is organized as follows: the developed system model based on PTD is presented in Section 2. It is shown that it relies on a generic reciprocity-based measurement model used in CIVA. Then the PTD-based system model is described first for the semiinfinite crack, then extended for a finite crack and then its extensions are discussed to regions surrounding the critical rays. Numerical validation of the model is discussed in Section 3 and experimental validation, in Section 4 for both pulse echo and TOFD configurations. Conclusions are presented in Section 5.

\section{Theory: modelling of scattering by cracks using a PTD based system model}

In this section, the developed system model based on PTD is presented. It is based on a generic reciprocity-based measurement model included in CIVA software which allows to account for both radiation from the emitting transducer and propagation of the radiated beam, scattering from flaws and reception of the scattered waves by the receiving transducer. PTD, which simulates only scattering from crack-like flaws, is incorporated into this CIVA generic measurement model to completely simulate an ultrasonic inspection of such defects. The elastodynamic PTD scattering model is then presented for a semi-infinite crack and its extension to a flaw of finite extent is shown afterwards. Some improvements of the model near critical observation angles are also proposed for shear scattered waves.

\subsection{A generic reciprocity-based measurement model}

The developed model relies on a generic system model [2], based on the reciprocity principle, which is employed in the CIVA software package [18]. CIVA is an expertise platform, developed by CEA-LIST and partners, for NDT simulation [19] and processing NDT data; its generic platform [20] allows to gather and add in the same software modelling tools of different nature and to compare their simulation results for a numerical validation purpose. The CIVA generic ultrasonic inspection model can be decomposed into three main modelling steps, the radiated beam model, the flaw scattering and the reception accounted by reciprocity [2].

The beam model is based on a semi-analytical method, which calculates the impulse response of the transmitting probe, assuming elementary sources distributed over the radiating surface. For each observation point, the contribution of each elementary source is then evaluated using an elastodynamic version of the pencil method [21]. This model can be used to compute the ultrasonic field inside a component radiated by probes of arbitrary shape, whether wedge coupled or immersed, whether monolithic or phased-arrays. Initially developed for homogeneous and isotropic materials, it has been extended to deal with anisotropic and heterogeneous media as well [22].

Depending on the nature of the flaw, different approaches are used in CIVA to model its interaction with the beam. Three classical scattering models are used, analytical approximations (such as the modified Born approximation for solid inclusions [23]), exact analytical solutions (such as the ones obtained for cylindrical or spherical cavities using the separation of variables method -SOV- [5]) and numerical solutions (such as the hybrid CIVA-ATHENA scheme, which relies on the finite elements method around the flaw). Crack echoes can be simulated until now using semianalytical models, such as KA [5] and GTD [10], or CIVA-ATHENA [24]. The latter relies on the pencil method [21] to simulate most of the propagation, while intricate interaction phenomena taking place in a small region surrounding the defects are computed using a FEM code ATHENA, developed at Electricité de France (EDF) in collaboration with the French National Institute for Research in Computer Science and Control (INRIA). In the system model proposed here, the flaw scattering from cracks is modelled using PTD.

The CIVA reciprocity-based measurement model and its abilities are described in more details in [2]. In order to avoid the electroacoustic transduction modelling, it requires as a modelling input the definition of a reference signal obtained by a calibration measurement on a reference flaw. Thanks to this calibration, the entire bandwidth of the transducers is taken into account in the echo calculations.

\subsection{Integration in CIVA of analytical scattering models (among them PTD)}

The different scattering models that were available in CIVA before the integration of PTD are listed in the previous section. As shown in [2], CIVA can employ different scattering models, the used scattering model being interchangeable. One advantage of the PTD model implementation is that it re-uses existing codes from the Kirchhoff and GTD models.

When integrating an analytical scattering model, two approximations or hypotheses are made [2]. The first one is the "local plane wave approximation" of the incident beam. The incident wave-field calculated by the CIVA beam model is approximated on each point of the flaw mesh by a local plane wave. The wave vector and polarization of this local plane wave as well as its 
amplitude are extracted from the CIVA beam calculation based on the pencil method. Consequently at each flaw meshed point, although the CIVA beam model accounts for all incident beam directions, only an average beam direction is involved for calculating the flaw scattering using the CIVA analytical scattering models. This approximation is usually valid in far field of the emitter or in its focal area if it is focused.

The second hypothesis is that the waves scattered from the mesh flaw points are spherical waves. It is the case for Kirchhoff approximation and also for GTD on finite flaws when coupling it to the Huygens' incremental method (described in Section 2.3); consequently, it is also the case for PTD which mixes Kirchhoff and GTD (see next section).

\subsection{The PTD based system model for a half-plane crack}

As explained in the Introduction, choosing between KA and GTD requires expertise. Moreover, in some configurations, neither model is entirely satisfactory. The PTD-based system model developed below overcomes the limitations of both. Indeed, in the PTD formalism [17], the Kirchhoff scattered field $\mathbf{u}^{\mathrm{KA}}(\mathbf{x})$ is evaluated in the far field using the leading order asymptotics. In this approximation the Kirchhoff scattered field is decomposed into two components, a geometrical (reflected) field and KA edge diffracted field. In this section, for simplicity, the scatterer is as in [4], a semiinfinite half-plane crack (with only one straight edge). In the elastodynamic version of PTD developed in [17] all scattered modes are taken into account, including mode conversions on the flaw. The Kirchhoff diffraction displacement field at an observation point $\mathbf{x}$ has the same form as the GTD field but a different edge diffraction coefficient $D_{\alpha \beta}^{K A}[17]$,

$\mathbf{u}^{\mathrm{KA}(\mathrm{diff})}(\mathbf{x})=\sum_{\beta} D_{\alpha \beta}^{\mathrm{KA}}(\mathbf{x}) \frac{\mathrm{e}^{i k_{\beta} r}}{\sqrt{k_{\beta} L_{\beta}}} \mathbf{d}_{\beta}$,

where $\alpha$ is the incident mode $\mathrm{P}, \mathrm{SV}$ or SH and $\beta$ is the scattered mode $\mathrm{P}, \mathrm{SV}$ or $\mathrm{SH} ; k$ is the scattered wave number; and $r$ is the distance from the edge to the observation point along the diffracted ray on the classical Keller cone [4]. The diffraction coefficient characterizes the directivity of the Kirchhoff edge diffraction contribution.

PTD [17] corrects the Kirchhoff edge diffraction field using GTD, which is the leading order asymptotics of the exact solution of the canonical scattering problem from a half-plane:

$\mathbf{u}^{\mathrm{PTD}}(\mathbf{x})=\mathbf{u}^{\mathrm{KA}}(\mathbf{x})+\sum_{\beta}\left[D_{\alpha \beta}^{\mathrm{GTD}}(\mathbf{x})-D_{\alpha \beta}^{\mathrm{KA}}(\mathbf{x})\right] \frac{\mathrm{e}^{i k_{\beta} r}}{\sqrt{k_{\beta} L_{\beta}}} \mathbf{d}_{\beta}$.

Consequently, the PTD scattered field is the sum of the original Kirchhoff scattered field (involving no far field approximation) and a modified GTD field, in which the classical GTD coefficient $D_{\alpha \beta}^{\mathrm{GTD}}(\mathbf{x})$ [4] is replaced by the difference $D_{\alpha \beta}^{\mathrm{GTD}}(\mathbf{x})-D_{\alpha \beta}^{\mathrm{KA}}(\mathbf{x})$. Similarly to KA, the total PTD field is the sum of the incident field and PTD scattered field.

In the specular direction, KA scattered field $\mathbf{u}^{\mathrm{KA}}(\mathbf{x})$ is finite and provides a good description of reflection. The KA diffraction coefficient $D_{\alpha \beta}^{\mathrm{KA}}(\mathbf{x})$ (obtained using the far field approximation of the KA field) diverges in the same manner as the GTD edge diffraction coefficient $D_{\alpha \beta}^{\mathrm{GTD}}(\mathbf{x})$. In $D_{\alpha \beta}^{\mathrm{GTD}}(\mathbf{x})-D_{\alpha \beta}^{\mathrm{KA}}(\mathbf{x})$ the two singularities cancel and therefore this difference is finite. Consequently, the PTD field is spatially uniform and unlike GTD, has no singularity at the specular angles. The reasoning applies to the incident shadow boundary too and in that case, the KA describes the compensating field which cancels the incident field in its shadow zone.
Near specular observation angles, the KA scattered field dominates and therefore

$\mathbf{u}^{\mathrm{PTD}}(\mathbf{x}) \approx \mathbf{u}^{\mathrm{KA}}(\mathbf{x})$

Away from the specular observation angles, the edge diffraction effects dominate and therefore

$$
\begin{aligned}
\mathbf{u}^{\mathrm{KA}}(\mathbf{x}) & \approx \sum_{\beta} D_{\alpha \beta}^{\mathrm{KA}}(\mathbf{x}) \frac{\mathrm{e}^{i k_{\beta} r}}{\sqrt{k_{\beta} L_{\beta}}} \mathbf{d}_{\beta} \text { and } \mathbf{u}^{\mathrm{PTD}}(\mathbf{x}) \\
& \approx \sum_{\beta} D_{\alpha \beta}^{\mathrm{GTD}}(\mathbf{x}) \frac{\mathrm{e}^{i k_{\beta} r}}{\sqrt{k_{\beta} L_{\beta}}} \mathbf{d}_{\beta}=\mathbf{u}^{\mathrm{GTD}}(\mathbf{x}) .
\end{aligned}
$$

\subsection{The PTD based system model for a finite size crack}

In the previous section, the PTD model has been described for the case of a semi-infinite stress-free half-plane crack. The elastodynamic GTD is well-established for such a canonical flaw [4]. In the following the application to a finite size crack is shown.

The implementation of PTD requires calculating the sum of the two terms involved in (2). The first term, corresponding to the Kirchhoff scattered field, is computed for a finite crack by meshing its illuminated parts and evaluating the Kirchhoff surface integral over these parts numerically. On each meshed elementary planar surface, the classical tangent plane Kirchhoff approximation is applied.

The second term of (2) is a modified GTD diffraction field. A finite size crack has a contour constituted of edges of finite size. However, GTD provides a solution to diffraction from canonical geometries like a stress-free half-plane having an infinite edge. Incremental models have been developed before in elastodynamics [25] in order to take into consideration the finite extent of the edge and validated experimentally [25]. One previously developed incremental model, the method based on the Huygens's principle [25] is used here. The formula (2) (involving a cylindrical or conical diffracted wave from an infinite edge) can be extended to a finite crack by summing the contributions of spherical waves along the crack contour, locally approximated by a straight edge. The finite flaw contour is thus approximated and meshed into straight portions which, following the Huygens's method [25], scatter a spherical wave whose directivity is proportional to the GTD coefficient of the infinite half-plane extending the current straight portion.

Finally the PTD computation for a finite flaw requires an integration on its illuminated surface for its Kirchhoff term and integration on its contour for its GTD modified term.

The integrated PTD model uses a specific recipe for multifaceted or branched flaws. Such flaws have two kinds of edges: edges at free extremities which can be modelled as a half-plane edges, and edges at internal junctions between branches which are wedge edges. In the CIVA-integrated PTD model, only the diffraction echoes from the flaw free edges are calculated using the PTD scattering model; echoes from internal junctions of branched facets are not since they cannot be approached by halfplanes and since the proposed PTD model is based on the GTD solution for a half-plane. Therefore in the CIVA-integrated PTD model, internal junctions echoes are calculated by Kirchhoff. This approach could be improved by implementing solutions of canonical problems of wedge diffraction to calculate junction echoes.

The proposed system model has been extended to deal with multiple rebounds on the component surfaces as well as mode conversions.

\subsection{PTD extensions}

When simulating diffracted shear waves all approximations described above break down around critical rays, which separate 
regions supporting the head waves from the corresponding shadow zones. This is because none of them model the head waves correctly (see Section 3.1.2): KA does not model them, because it is based on the GE approximation of the surface field and in GE the head waves do not arise. GTD and therefore standard PTD fail too, because they model the critical zones well only in the very far field $[26,27]$. We propose two recipes to improve the PTD performance near the critical observation angles. These recipes consist in modifying the PTD model only at the vicinity of critical angles. Far from critical angles, they are identical to the PTD model.

The first recipe is based on the use of a mixed analytical and numerical model $[26,27]$ called PTD/Simpson. In [26,27], a modified Simpson's method has been developed to calculate numerically the exact integral solution of the canonical half-plane scattering problem $[26,27]$. The PTD/Simpson model operates as follows: when the stationary and branch points of the exact integral coalesce, that is, when edge diffracted waves and head waves interfere, the mixed PTD/Simpson model utilizes the modified Simpson's method, relying on the classical PTD otherwise.

The second recipe simply smoothes the GTD diffraction coefficients. To be precise, in the vicinity of the critical angle the magnitude of the GTD coefficient is interpolated linearly, while its phase is smoothed using the following smoothed Heaviside function:

$$
\begin{array}{ll}
H_{\text {smooth }}(x)=0 & \text { if } x<-h, \\
H_{\text {smooth }}(x)=0.5 *(1+x / h+\sin (p i * x / h) / p i) & \text { if }|x|<h, \\
H_{\text {smooth }}(x)=1 & \text { if } x>h .
\end{array}
$$

where $h$ is chosen equal to $10^{\circ}$. It has been established by trial and error that this value of $h$ is a satisfactory compromise, affecting the value of the coefficient on a small range of observation angles while allowing it to vary smoothly as a function of the observation angle.

\section{Numerical validation}

Numerical validation of the proposed models has been performed by comparing them with various known models in both pulse echo configuration and TOFD configuration in ferritic steel components. The hybrid CIVA/ATHENA [24] model has been used as a reference. It allows one to simulate the $2 \mathrm{D}$ response of SDHs and cracks (rectangular, 2D CAD, multifaceted and branched) by using both analytical models and finite elements simulations near the defects. The principle of this technique is the following. A 2D domain in the specimen is defined, embedding the defects. The field reaching the domain entry is calculated by the analytical CIVA pencil method on the domain border. Then the field is propagated in the defined domain by the finite elements scheme ATHENA which accounts for the beam to flaw interaction. The FEM domain includes the flaws whose geometry is accounted in an optimized way (notably for complex-shaped defects) by the ATHENA FEM codes using the fictitious domains method [24,28,29]. The extent of the FEM domain is chosen so that its boundary are enough far from the defects (at least at one wavelength) to take into account correctly the radiation of surface and head waves from the flaws. The time and spatial steps of the numerical scheme are linked by the Courant-Friedrichs-Lewy (CFL) condition with a CFL number equal to 1 . The steps of the spatial discretization are chosen to ensure at least 13 mesh points per wavelength of the slowest shear wave at the highest frequency of the signal.

In this section, we intend to validate the PTD models. In a GTD or PTD solution, a semi-infinite half-plane is considered and the crack faces are assumed to be stress-free and non-interacting, i.e. the crack remains open under the influence of the incident wave (see [30]). The crack is supposed consequently to have at his edges an aperture which is quasi-null (but not null to avoid interaction of its faces). The crack is consequently modelled as infinitely narrow at his edges in FEM calculations in order to reproduce the PTD assumptions, for numerical validation purposes. If the crack has a thin aperture at one edge, GTD and PTD are not theoretically applicable but the coupling code CIVA/ATHENA 2D allows taking into account the real edge aperture and has been shown to lead to a good agreement with experiments in TOFD configurations [31].

In the following, FEM refers to the hybrid code CIVA/ATHENA.

\subsection{Pulse echo configuration}

\subsubsection{SV waves}

The first typical pulse echo configuration studied is presented in Fig. 1a. It involves SV45 ${ }^{\circ}$ waves at $5 \mathrm{MHz}$ and $5 \mathrm{~mm}$ high defects of an arbitrary tilt angle $\alpha$. A $12.7 \mathrm{~mm}$ diameter probe is used in immersion with a $20 \mathrm{~mm}$ water path. A defect is located inside the area of maximum field amplitude, which corresponds to depths between $33 \mathrm{~mm}$ and $40 \mathrm{~mm}$.

In Fig. 2, GTD, KA and PTD are used to simulate the echo amplitude (in $\mathrm{dB}$ ) as a function of the tilt angle $\alpha$. The tilt angle is measured with respect to the vertical direction. The results illustrate the unifying nature of the system model based on PTD. Indeed, when the probe detects the signal reflected by the flaw (around $\alpha=-45^{\circ}$ in Fig. 1b, also see the yellow ${ }^{1}$ area in Fig. 2 ), GTD is invalid but KA produces good quality results. The system model based on PTD produces the same results as the model based on KA. When the probe detects the diffracted signal (see the grey areas in Fig. 2), KA does not perform very well. To give one example, in the classical case of a vertical flaw inspected with the $S 45^{\circ}$ wave (for $\alpha=0^{\circ}$, see Fig. 1c), the KA error compared to FEM is $5 \mathrm{~dB}$ (see Fig. 3). By contrast, GTD performs effective. In the grey areas, PTD gives the same results as GTD.

In Fig. 3, the configuration is the same as in Fig. 2, but the output of the 2D FEM CIVA/ATHENA code is added for additional comparison. The simulated amplitudes shown in Fig. 3a are absolute (not in dBs as in Fig. 2) and Fig. 3b zooms in on small amplitudes in Fig. 3a. Fig. 3a demonstrates a perfect agreement between PTD and FEM in the region around $\alpha=-45^{\circ}$, where the scatter is near specular and therefore the signal received by the probe is due to the reflected echo. When the received signal is due to a diffracted $S$ wave the predictions of the model based on PTD model can lead to some prediction errors in diffraction compared to the model based on FEM (Fig. 3b). Indeed, above the critical angle the FEM curve oscillates due to interference between the head wave (see Fig. 4 below) and the $S \rightarrow S$ diffracted wave. The PTD based model does not account for this interference. This oscillation behaviour is manifest in steel for the observation angles greater than the critical angle $33^{\circ}$. The typical PTD error is acceptable in NDT applications except when the angle is near critical $\left(\alpha=-78^{\circ}\right.$ or $\left.-12^{\circ}\right)$. When the flaw height increases, the oscillations fade and the quality of the PTD simulation is improved.

Fig. 4 presents the paths of the diffracted waves generated at critical incidence by the bottom tip of the backwall breaking crack and comprise (a) a classical tip-diffracted SV bulk wave, (b) S wave shed in the backscattering direction by the bottom tip irradiated by a creeping P wave (some authors call the latter the P component of the head wave), (c) the head wave (some authors call it the S component of the head wave), also shed in the backscattering direction. At critical angles both GTD and PTD amplitudes exhibit peaks (Fig. 3b) and around the critical angles simulations of SV waves are less reliable (particularly for small flaws).

To understand the deterioration in the quality of PTD near critical angles, the $45^{\circ} \mathrm{SV}$ oblique incidence is analysed for a vertical

\footnotetext{
${ }^{1}$ For interpretation of color in Figs. 2 and 5, the reader is referred to the web version of this article.
} 


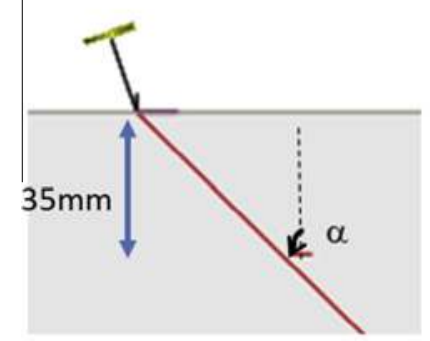

(a)

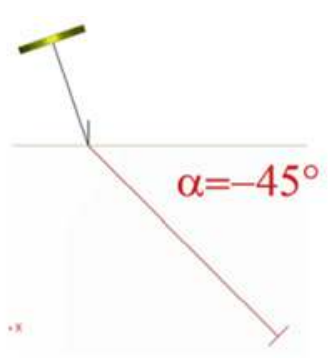

(b)

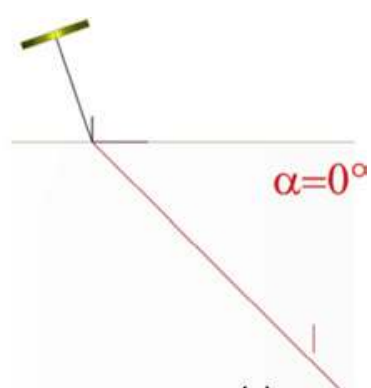

(c)

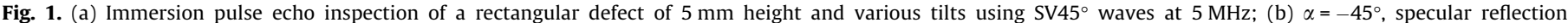
configuration; (c) $\alpha=0^{\circ}$, the classical case of a vertical flaw inspected with the $S 45^{\circ}$ wave in pulse echo configuration.

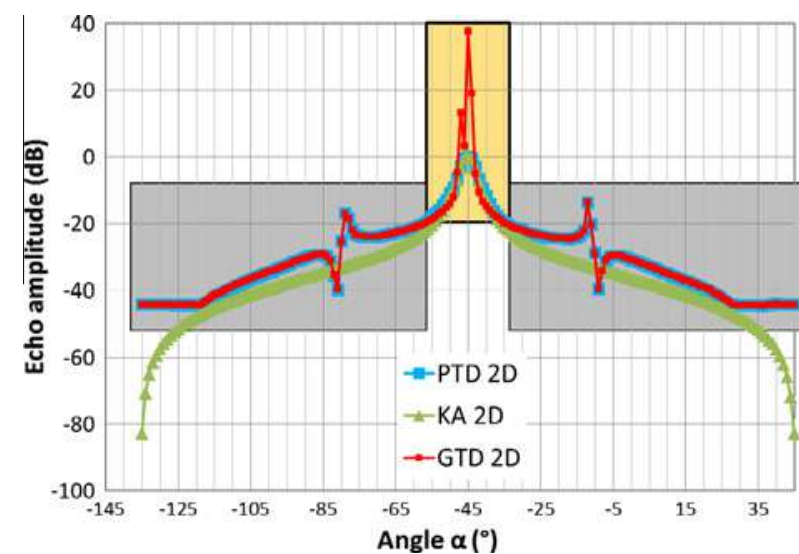

Fig. 2. Numerical validation of PTD in the pulse echo configuration of Fig. 1. Echo magnitudes (in $\mathrm{dB}$ ) versus the tilt angle $\alpha$, simulated using GTD, KA and PTD.

flaw of $5 \mathrm{~mm}$ height using snapshots of the ultrasonic field simulated using FEM (see Fig. 5). In these snapshots $S_{1}$ is the wave front of the incident beam. The geometrical axes of the incident and reflected $S$ beams are shown in red. $S_{3}$ is the single wave reflected on the flaw. At the angles larger than critical there are no $P$ reflected waves. $\mathrm{P}_{1}\left(\mathrm{~S}_{2}\right)$ and $\mathrm{P}_{2}\left(\mathrm{~S}_{4}\right)$ are the wave fronts of the waves diffracted by the top and bottom crack tips, which have undergone (no) mode conversion. In (c), (d) and (e) a scattered Rayleigh wave is observed, which is due to the secondary diffraction (see Fig. 6). When the beam hits the top (bottom) tip, the Rayleigh wave $R_{1}$ $\left(R_{2}\right)$ is generated. This propagates along the crack face towards the opposite tip. On reaching the bottom tip $R_{1}$ is "reflected" to produce $R_{3}$ and sheds the bulk $S_{5}$ wave. At the top tip, $R_{2}\left(R_{3}\right)$ generates the $S_{6}\left(S_{7}\right)$ bulk diffracted wave.

In snapshots (b), and particularly (c) of Fig. 5, behind the crack, in the shadowed region not irradiated by the incident beam one can see the straight front of the head wave (both the rays carrying the head wave and its front are drawn in yellow). The line connects the fronts of $P_{1}$ and $S_{2}$ cylindrical waves diffracted by the top crack tip. It is tangential to the $S_{2}$ front. The path of the head wave is similar to that depicted in Fig. 4c.

After a study based on numerical validation using CIVA/ ATHENA, whereas the PTD prediction of specular reflection is shown to be valid for direct SV waves echoes for ka > 1.5 about, the validity of edge diffraction prediction by PTD appears to be $\mathrm{ka}>(\mathrm{ka})_{\max }$ with $(\mathrm{ka})_{\max } \in[5,10]$ depending on the tilt angle and on the distance between the flaw and the probe (since head waves attenuate with this distance).

\subsubsection{Regions surrounding critical rays}

Let us now validate against FEM the two extensions of PTD proposed for simulating the regions surrounding the critical rays. We revisit the pulse echo inspection configuration of Fig. 1 and consider two flaws, one $5 \mathrm{~mm}$ high and another, $20 \mathrm{~mm}$ high. As above, both crack centres are located at the depth of $35 \mathrm{~mm}$. The echo amplitudes are simulated in Fig. 7 using the classical PTD, PTD with smoothing, PTD/Simpson and CIVA/Athena FEM method.

As discussed in [26], when using the PTD/Simpson model, in the near field, that is, at small distances from a half-plane flaw the critical spikes in GTD (or PTD) scattering coefficients are smoothed. The same effect is observed in Fig. 7. It is due to destructive interference between the head waves and diffracted waves and

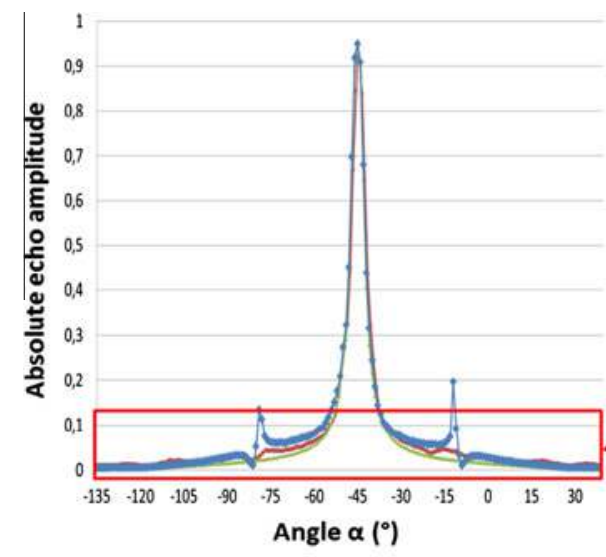

(a)

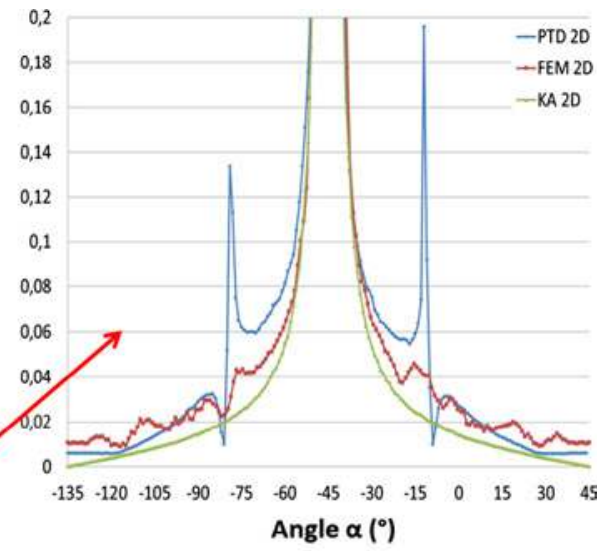

(b)

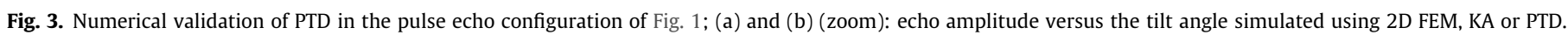




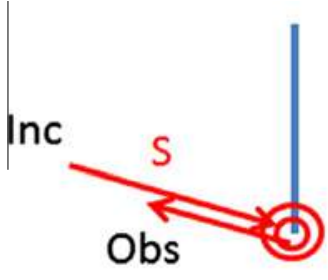

(a)

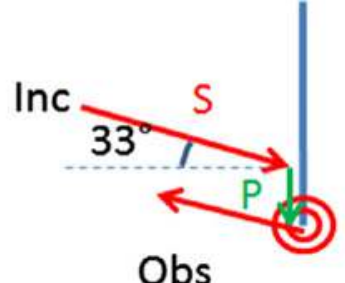

(b)

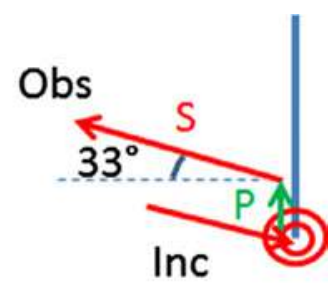

(c)

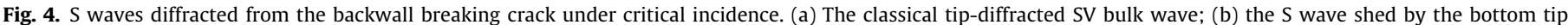
irradiated by the P creeping wave; (c) the head wave radiated after diffraction of a P creeping wave at the bottom tip.
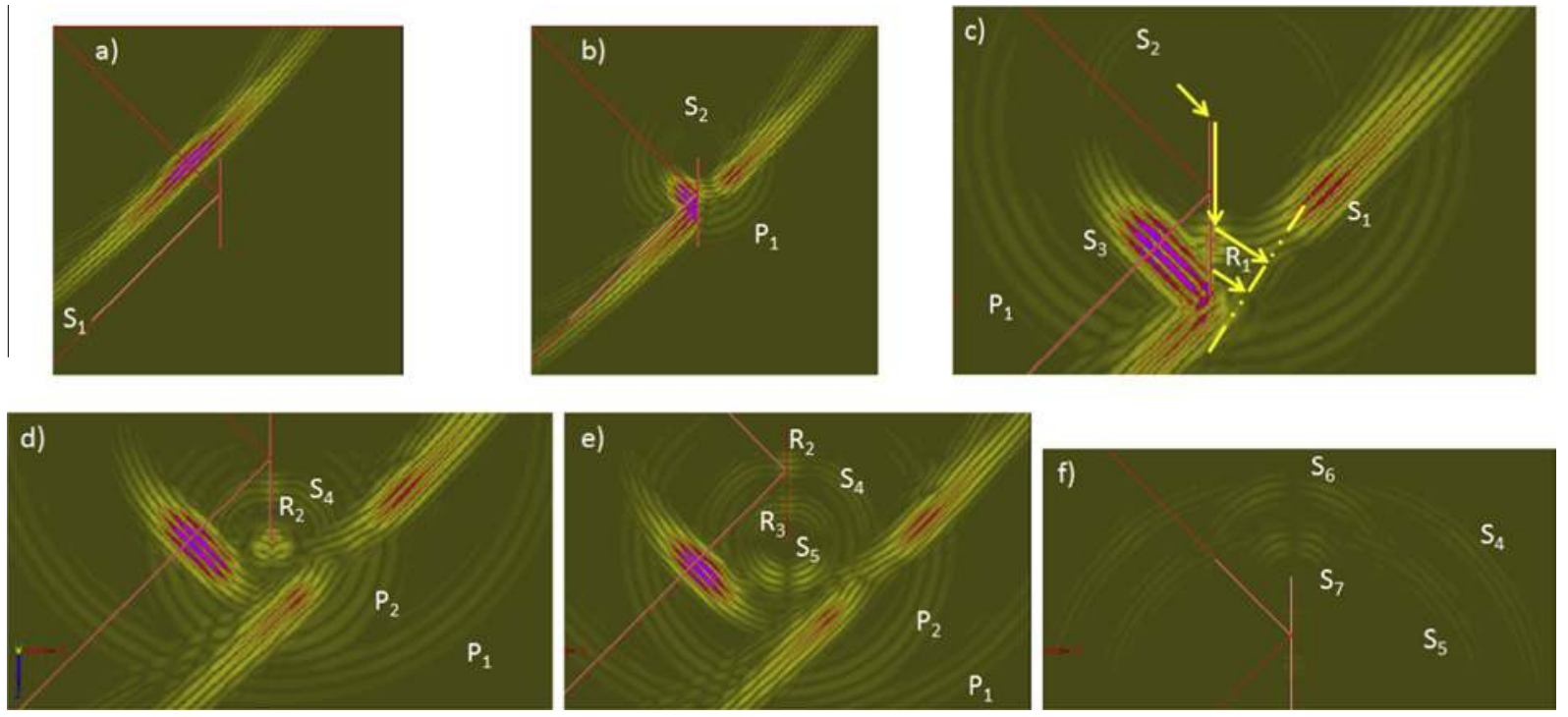

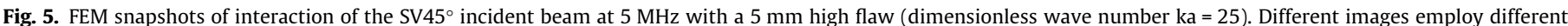
scales.

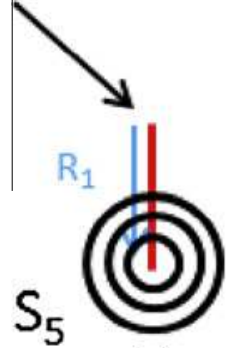

(a)

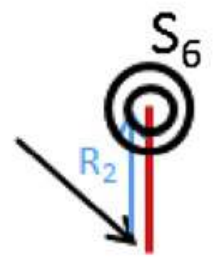

(b)

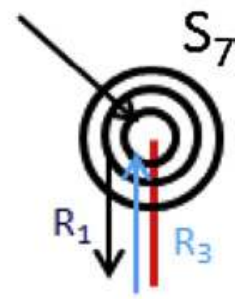

(c)

Fig. 6. Wave fronts of the waves observed in Fig. 5. (a) The top to bottom Rayleigh wave path $R_{1}$ and wave front $S_{5}$ of the diffracted wave it generates, (b) the bottom to top Rayleigh wave path $R_{2}$ and wave front $S_{6}$ of the diffracted wave it generates, (c) the wave $R_{3}$ reflected at the bottom tip producing the $S_{7}$ wave.

disappears away from the edge, since the head waves attenuate with the distance faster than the classical diffracted waves. For the same reason, at supercritical angles $(\alpha>-8)$, the PTD/Simpson amplitudes oscillate. The oscillations are less marked for the $20 \mathrm{~mm}$ high flaw, because the head wave attenuates as it propagates along the flaw. Nevertheless, the PTD/Simpson results are significantly different to the FEM results, which are perfectly smooth. This is due to the fact that the PTD/Simpson has been developed for a semi-infinite and not a finite crack.

This is confirmed further by mimicking the situation of an infinite half-plane and simulating for that purpose a pulse echo

inspection configuration similar to that in Fig. 1 but with a very large flaw located in the very far field, its edge being at the depth of $165 \mathrm{~mm}$ (see Fig. 8a). Contrary to the previous example (Fig. 7), the PTD/Simpson spikes (Fig. 8b) are similar to the GTD/PTD ones, since head waves attenuate with the distance to the edge. Unlike the flaws of the $5 \mathrm{~mm}$ or $20 \mathrm{~mm}$ height in Fig. 7, the FEM results (Fig. 7b) exhibit a spike similar to the GTD one too, which confirms that the latter are physical in nature for a semi-infinite flaw. However, the FEM spike is smaller. This may be due to the fact that the FEM model accounts for all incident beam directions, whereas in PTD only an average beam direction is exploited at each edge point.

It follows that for smaller flaws such as those considered in Fig. 7, the FEM curve is smooth and the PTD smoothing model gives a much better recipe than the PTD/Simpson one. The smoothness of the FEM results is due to interference of head waves multiply reflected at crack tips. It is not surprising that such smoothing is not offered by PTD/GTD - no multiple reflection arises on semiinfinite flaws. Other authors have reported similar behaviour and justification for the smoothing of critical peaks in diffraction coefficients for finite strips [3,32].

It follows that the PTD smoothing model is suitable for modelling scattering by finite flaws common in NDT applications and critical observation angles when head waves interfere with edge diffracted waves. However, the model is not suitable for simulating inspection configurations involving critical incidence, because in such cases, the head waves interfere with reflected waves and KA breaks down. 


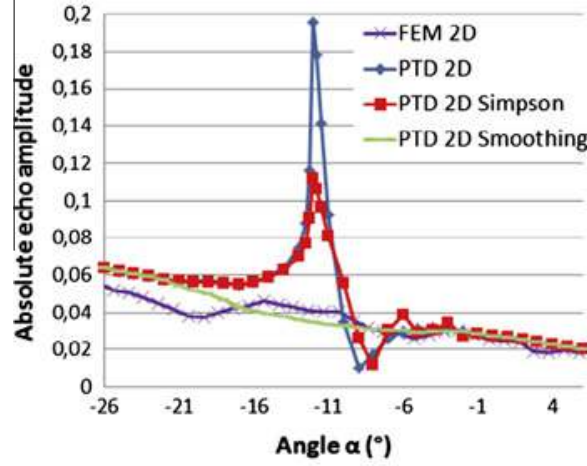

(a)

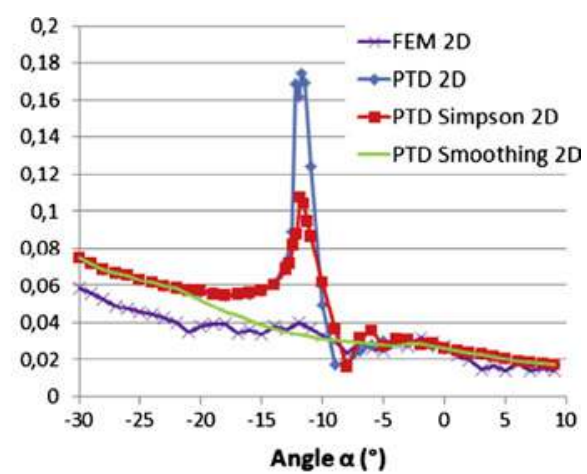

(b)

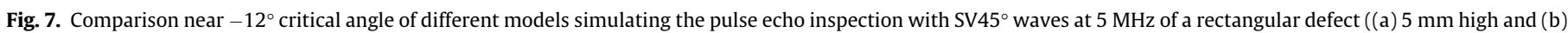
$20 \mathrm{~mm}$ high) of various tilt.

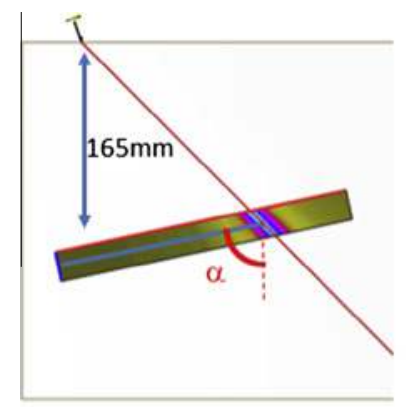

(a)

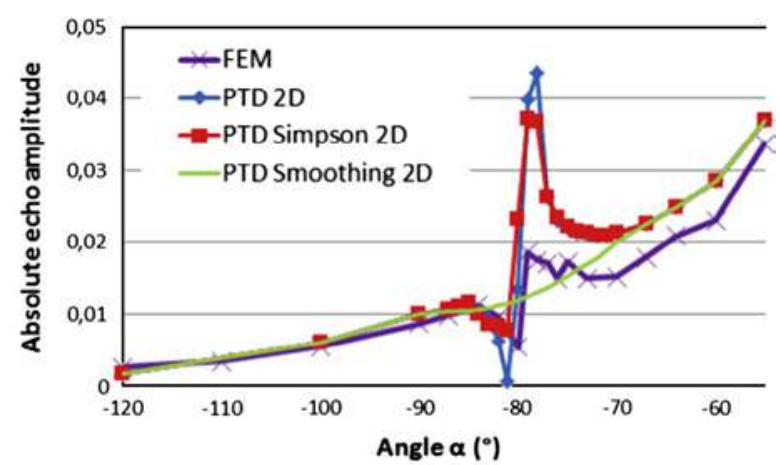

(b)

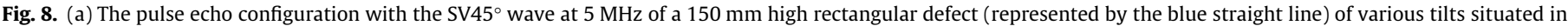

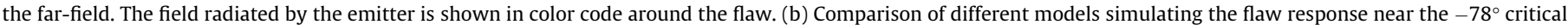
angle. (For interpretation of the references to colour in this figure legend, the reader is referred to the web version of this article.)

\subsubsection{P waves}

The next series of numerical experiment involves the pulse echo inspection with incident $\mathrm{P}$ waves at $5 \mathrm{MHz}$ of a rectangular defect of $5 \mathrm{~mm}$ height and various tilts. A cylindrical specimen is used and the probe emitting $\mathrm{PO}^{\circ}$ waves is rotated to be positioned so that the incident direction is normal to the component surface (Fig. 9a). During this type of scanning, the beam impinges on the flaw with $P$ waves at different angles of incidence. The procedure has the same effect as varying the flaw tilt angle in Fig. 1. In Fig. 9b the echo amplitude (in $\mathrm{dB}$ ) is simulated with different 2D models: CIVA/ATHENA (FEM), KA and PTD are plotted versus the observation angle $\alpha$. Fig. 9c is a zoom of Fig. 9b at small amplitudes. The agreement of PTD model with FEM is very good for all tilt angles. As expected, a good agreement with FEM is achieved by KA near the reflection angle $\alpha=90^{\circ}$ (see Fig. 9b) and by GTD (not reproduced for simplicity) in diffraction configurations. In the latter case, for the tilt angles, which are far from specular, KA produces significant prediction errors.

For both $\mathrm{P}$ and SV waves, a deterioration in the PTD predictions is observed for small flaws (rarely encountered in NDT) in TOFD configurations, particularly because the Rayleigh waves propagating along the defect are not described by classical PTD. For P waves, the overall validity range of PTD $\mathrm{ka}>(\mathrm{ka})_{\max }$ is about (ka) $)_{\max } \in[1,3]$; the limit is much lower than for SV waves.

To understand the difficulties arising in modelling small flaws further, we consider a typical case of the $\mathrm{P} 45^{\circ}$ oblique incidence (at $5 \mathrm{MHz}$ ) on a $2 \mathrm{~mm}$ high flaw and analyse its backscattering response using FEM snapshots (Fig. 10). This figure is more difficult to interpret than Fig. 5, because of multiple mode conversions. $P_{1}$ is the wave front of the incident beam arriving from the bottom of the figure. The geometrical axes of the incident and reflected beams are drawn in green. $\mathrm{P}_{2}$ is the reflected wave. $\mathrm{P}_{3}$ and $\mathrm{P}_{4}$ are the classical bulk waves diffracted from, respectively, the bottom and top crack tip. In the far field, $\mathrm{P}_{1}, \mathrm{P}_{3}$ and $\mathrm{P}_{4}$ all merge behind the flaw, at the shadow boundary of the incident wave. Behind the flaw, the incident field is small but not negligible: The incident beam is almost a plane wave and the incident rays are intercepted by the flaw, but the resulting diffracted rays $\left(\mathrm{P}_{3}\right.$ and $\left.\mathrm{P}_{4}\right)$ penetrate the region. In the area insonified by the incident beam, $\mathrm{P}_{3}$ and $\mathrm{P}_{4}$ merge with $\mathrm{P}_{2}$ at each shadow boundary of the reflected $\mathrm{P}$ field. $S_{1}$ is the $S$ wave reflected (mode converted) by the flaw, and $S_{2}$ and $S_{3}$ are the classical mode converted bulk waves diffracted from, respectively, the bottom and top crack tip. $S_{2}$ or $S_{3}$ merge with $S_{1}$ at each shadow boundary of the reflected $S$ (mode converted) field.

In snapshots (c) and (d) the secondary diffractions are observed, which are elucidated in Fig. 11. It shows that $S_{4}$ and $P_{5}$ are generated by the top tip diffracting the Rayleigh wave that is generated by the bottom tip and then propagates along the flaw surface upward. $S_{5}$ and $P_{6}$ (not shown) are later contributions due to the top to bottom Rayleigh wave. In the snapshot in Fig. 11a, one can observe the corresponding SV secondary diffractions $\left(S_{4}\right.$ and $\left.S_{5}\right)$, which have a larger amplitude than the mode converted secondary P diffracted waves. Since the Rayleigh wave speed is close to the SV 


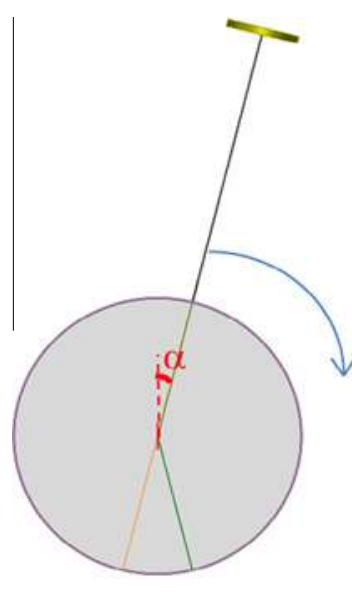

(a)

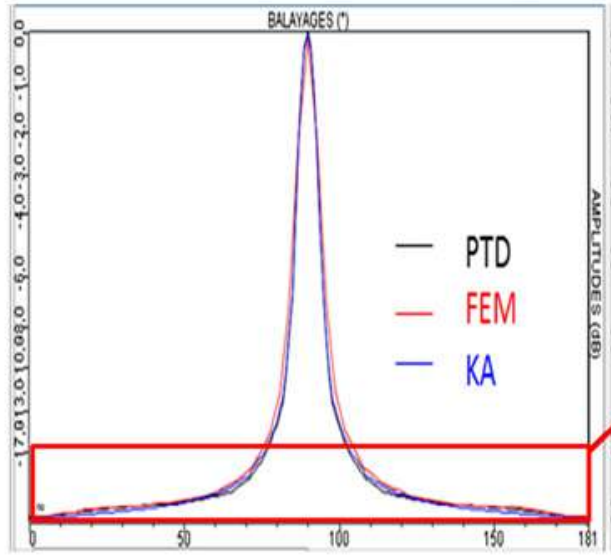

(b)

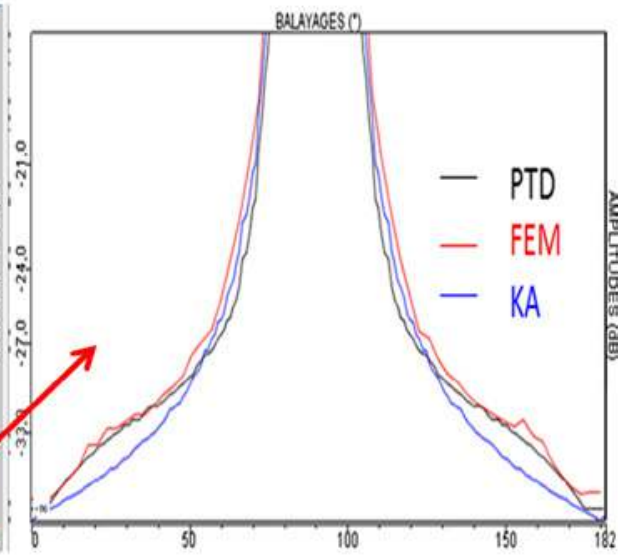

(c)

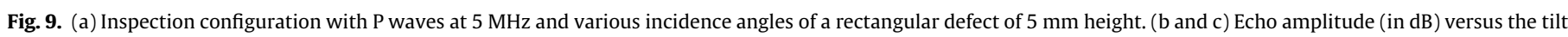
angle (in ${ }^{\circ}$ ) simulated with 2D FEM, KA and PTD.
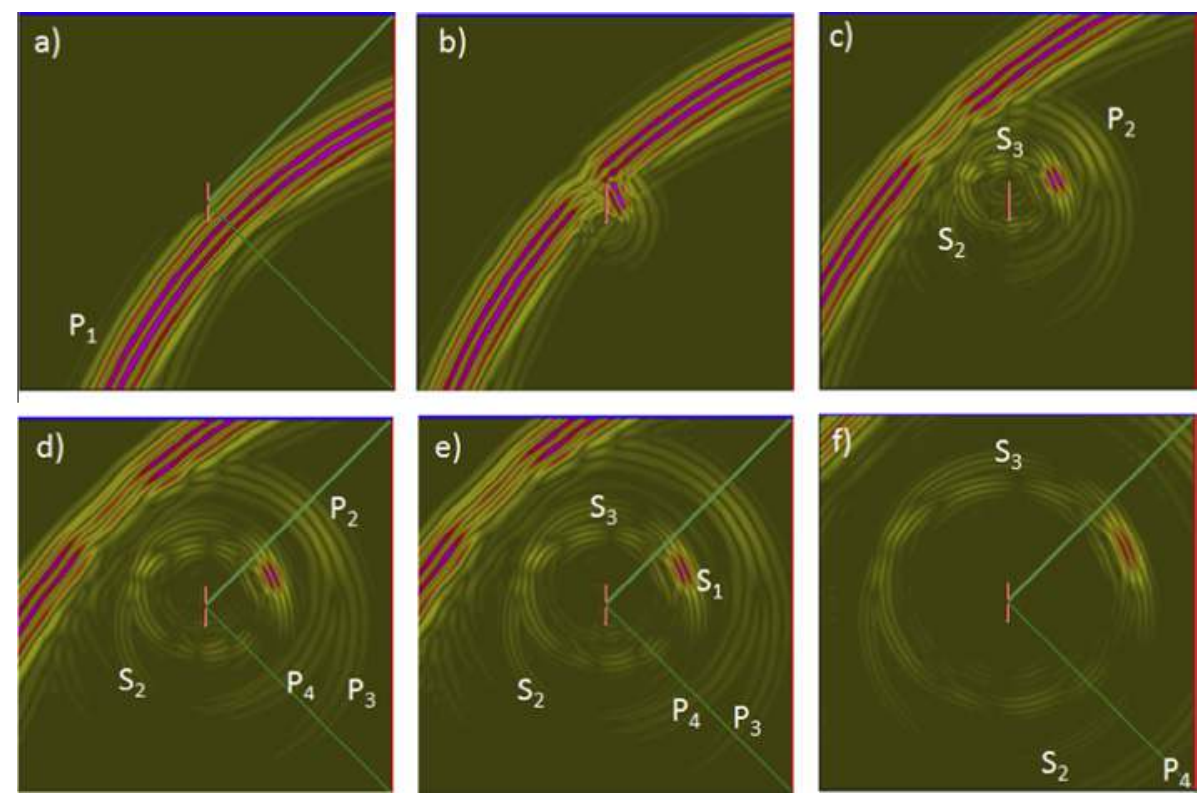

Fig. 10. FEM snapshots of interaction of the $\mathrm{P} 45^{\circ}$ incident beam at $5 \mathrm{MHz}$ with a $2 \mathrm{~mm}$ high flaw (dimensionless wave number ka $=5$ ).

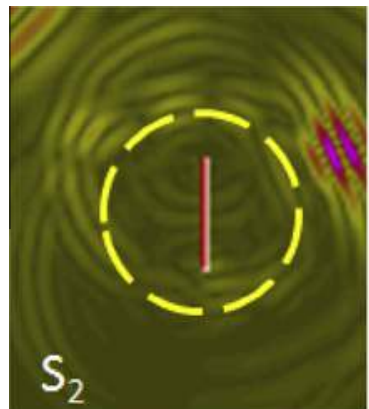

(a)

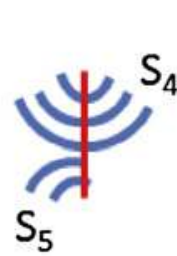

(b)

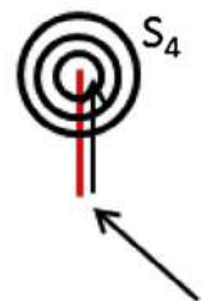

(c)

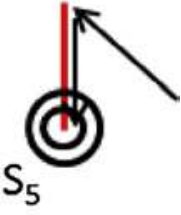

(d)

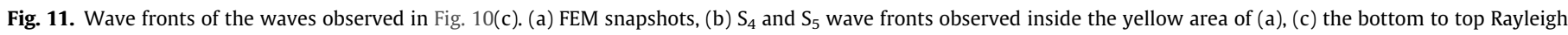

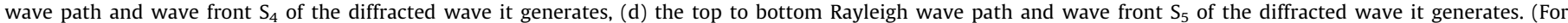
interpretation of the references to colour in this figure legend, the reader is referred to the web version of this article.) 
speed, $\mathrm{S}_{4}$ starts being diffracted by the top tip about the time it is reached by the wave front of $S_{2}$ (Fig. 11a), which has been originally diffracted by the bottom tip.

\subsection{TOFD configuration}

A typical TOFD configuration presented in Fig. 12a involves the SV45 ${ }^{\circ}$ wave at $5 \mathrm{MHz}$ and a $5 \mathrm{~mm}$ high rectangular defect at various tilt angles $\alpha$. To study it two identical probes $(12.7 \mathrm{~mm}$ diameter) have been used in immersion, with $20 \mathrm{~mm}$ water path and $85 \mathrm{~mm}$ Probe Center Separation. The flaw is located near the crossing point of the two probes' beams. Fig. 12b presents the flaw echo amplitude versus the tilt angle $\alpha$ simulated with GTD, KA, classical PTD and PTD with smoothing. In this configuration, the critical tilt angles are $-102^{\circ},-78^{\circ},-12^{\circ}$ and $12^{\circ}$, but at these angles the discontinuities in the PTD echo amplitudes prove small or negligible. Therefore the smoothed and non-smoothed PTD models produce similar results. A particularly good agreement between PTD and FEM is obtained in Fig. 12b near the specular direction $\alpha=-90^{\circ}$. However, for the scattered S waves, particularly, near the critical angles PTD does not perform as well as FEM. Still, the overall agreement is satisfactory. Note that similarly to the pulse echo experiments for SV waves, errors in predicting the diffracted waves with KA are significant. Therefore, so should be the errors in the Huygens-Fresnel method of [11], which relies on the Kirchhoff integral over a virtual flaw.

Thus, the model based on PTD with smoothing performs well in both pulse-echo and TOFD configurations.

\section{Experimental validation}

Experimental validation of the system model based on PTD with smoothing has been carried out in both pulse echo and TOFD configurations by studying corner echoes resulting from reflections and diffraction. This is part of a CIVA experimental validation campaign which has been carried out for several years [33].

\subsection{Pulse echo configuration: corner echoes}

In order to validate simulation using PTD model with smoothing of corner echoes in pulse echo configuration, measurements have been performed on a mock-up with vertical backwall breaking notches of various height (Fig. 13a). A 64 elements contact matrix phased-array probe with a wedge generating $45^{\circ}$ compressional waves at $2.15 \mathrm{MHz}$ (60\% bandwidth) has been used
(Fig. 13c): its total dimensions is $446 * 20 \mathrm{~mm}^{2}$ with a gap between elements of $0.2 \mathrm{~mm}$ in both directions. 2D scanning has been performed over each reflector. At each probe position, several focusing depths (from 20 to $40 \mathrm{~mm}$ depth) have been employed, with the $\mathrm{P} 45^{\circ}$ deviation. The reference amplitude is the maximum amplitude of the specular $\mathrm{P} 45^{\circ}$ direct echo from a $2 \mathrm{~mm}$ diameter SDH positioned in the calibration mock-up at the $72 \mathrm{~mm}$ depth. To check their reproducibility, all measurements have been carried out several times (minimum two times for each flaw). The maximum difference in the results has proved to be less than $1 \mathrm{~dB}$. Fig. 13c and d shows experimental and simulated reconstructed B-scans obtained over the $15 \mathrm{~mm}$ high backwall breaking notch with the $\mathrm{P} 45^{\circ}$ deviation and focusing depth of $30 \mathrm{~mm}$. A good agreement between experimental and simulated B-scans and echodynamics has been obtained. The corner echo involving no mode conversion is identified as arriving from the bottom flaw tip in reconstructed B-scans. Some differences are observed in the $\mathrm{T}$ corner echoes, since the $\mathrm{T}$ beam includes head waves generated at the wedge/component interface, and these waves are not accounted for in the beam model. Note that in this case, the corner echo is due to two specular reflections, one from the backwall and one from the flaw. Consequently, PTD gives results similar to KA (Fig. 13b).

For all flaw heights and focusing depths, the resulting discrepancy in $\mathrm{P}$ corner echoes has proved to be around or less than $2 \mathrm{~dB}$ (see Fig. 14). The most significant differences appear for small notch heights when the P diffraction echo from the notch top edge is mixed with the $\mathrm{P}$ corner echo. These interferences explain the maximum in the plot of echo amplitude versus the notch height observed both experimentally (around $2 \mathrm{~mm}$ height) and in simulation (with a slight shift).

\subsection{TOFD configuration: edge diffracted echoes and 3D configurations}

In order to validate simulations of TOFD configuration [34], results presented in [30] are discussed first. The authors have employed a 2D symmetrical arrangement of the transmitting and receiving probes over a cylindrical mock-up containing a real fatigue crack (Fig. 15a) and investigated variation in the amplitude of the echo diffracted from the bottom flaw tip with the change in the orientation of the transmitted and received beams (Fig. 15b).

The simulated curve has a well-defined minimum at about $38^{\circ}$, which corresponds to the minimum of the P-P GTD coefficient [30]. There is a good agreement between the PTD based model

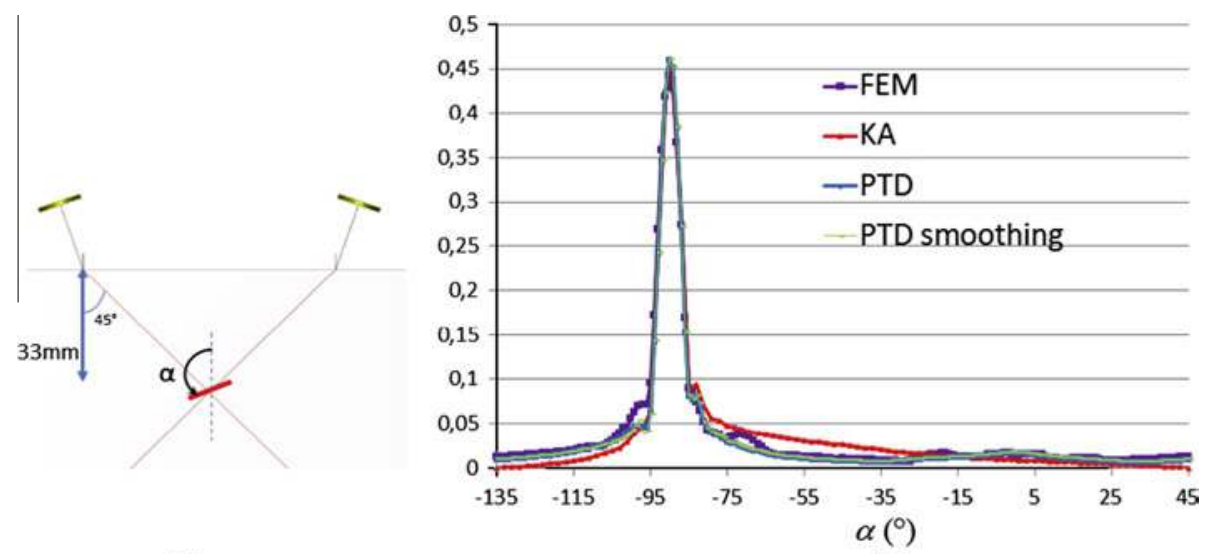

(a)

(b)

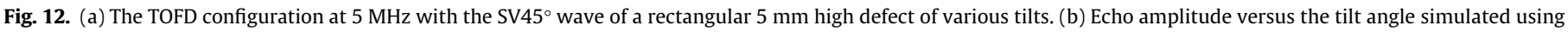
2D FEM, KA, PTD and PTD with smoothing around the critical angles. 


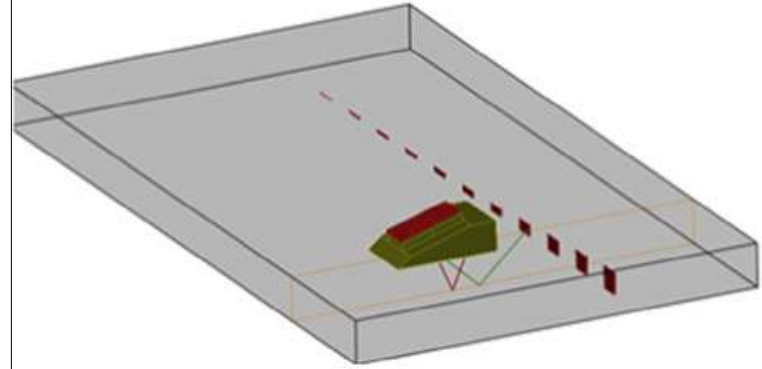

(a)

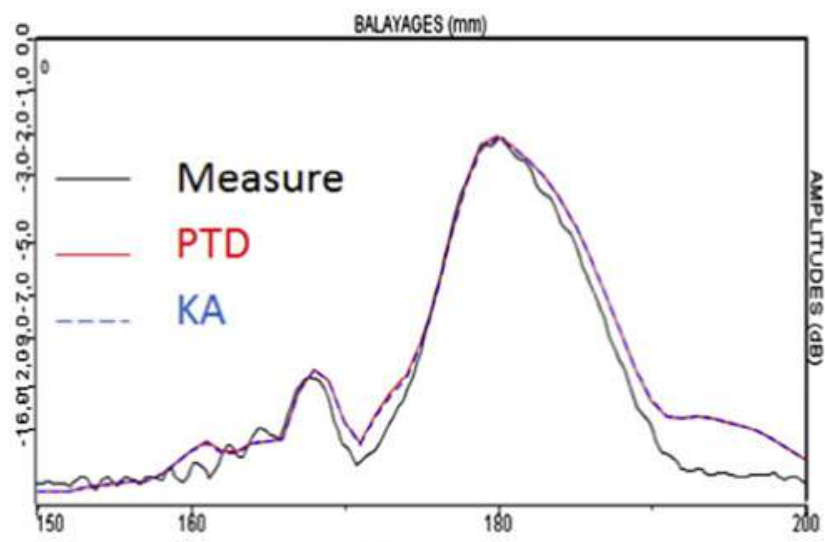

(c)

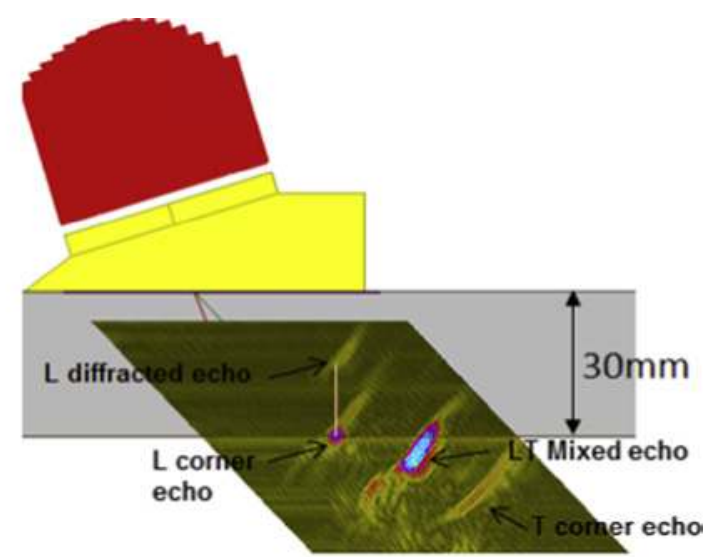

(b)

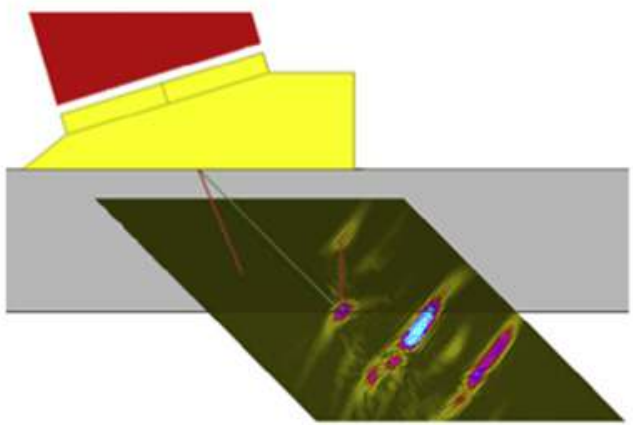

(d)

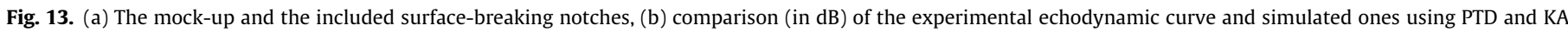

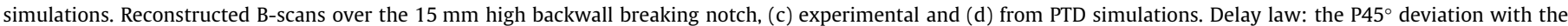
focusing depth of $30 \mathrm{~mm}$.

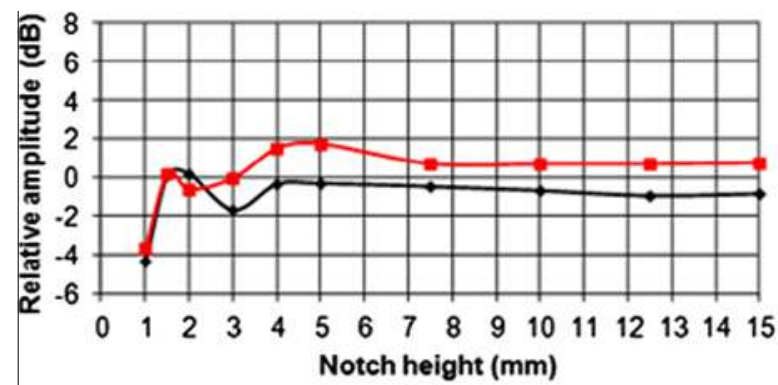

(a)

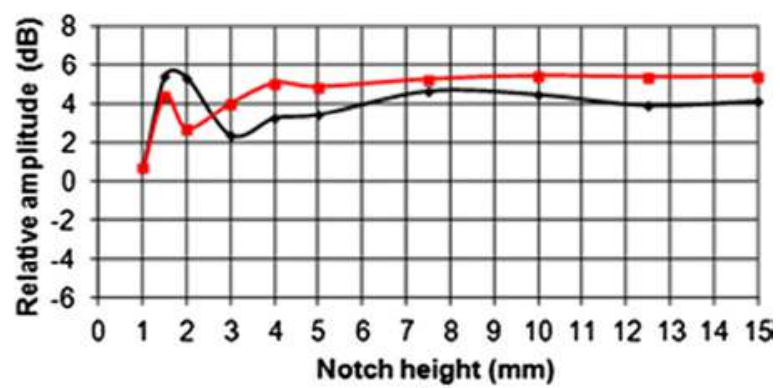

(b)

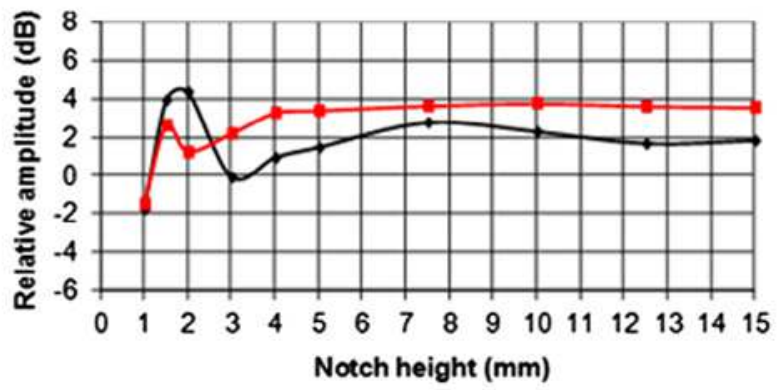

(c)

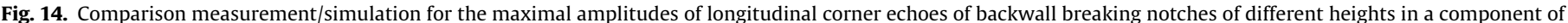

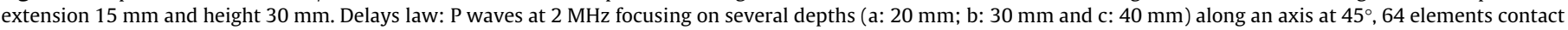
matrix probe. 


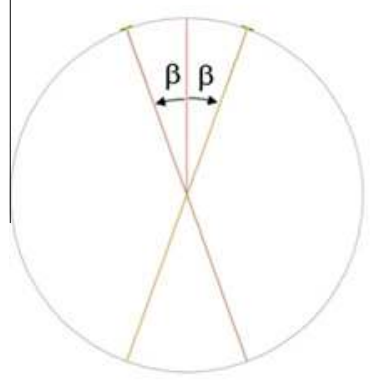

(a)

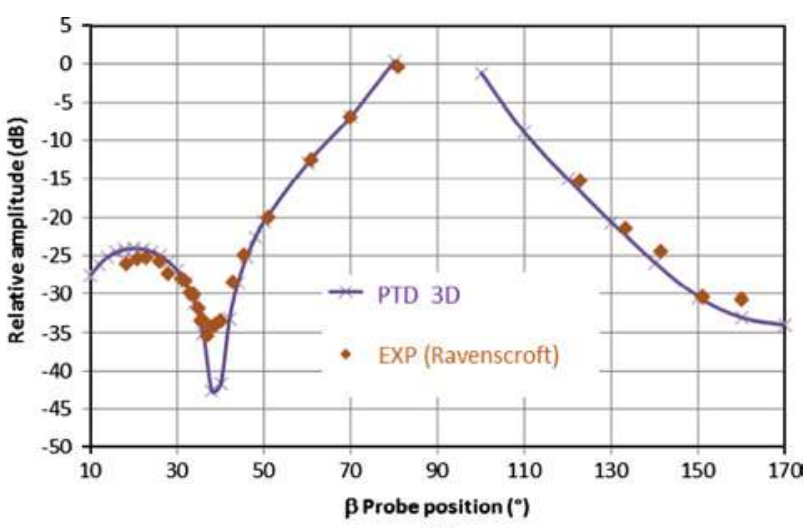

(b)

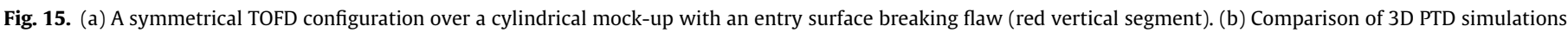

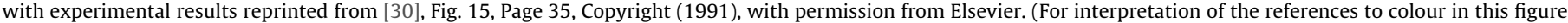
legend, the reader is referred to the web version of this article.)

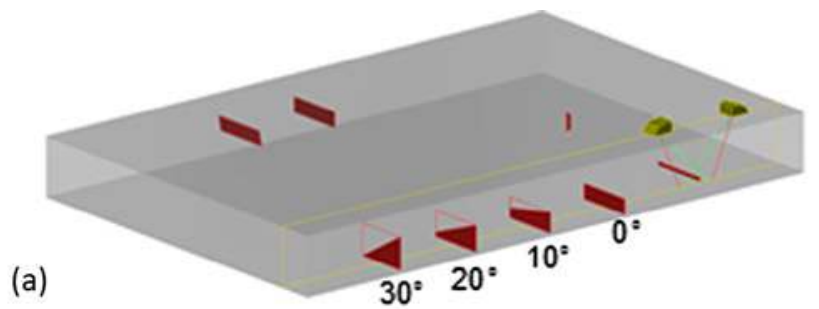

(b)

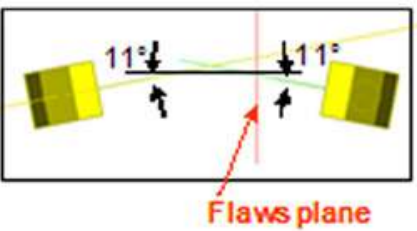

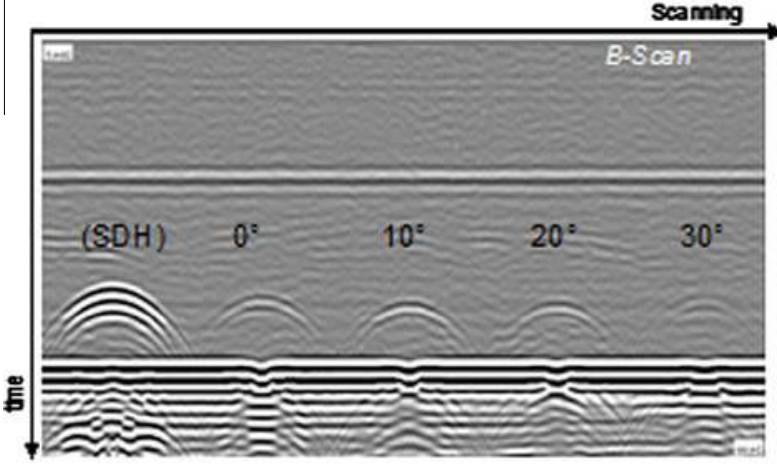

(c)

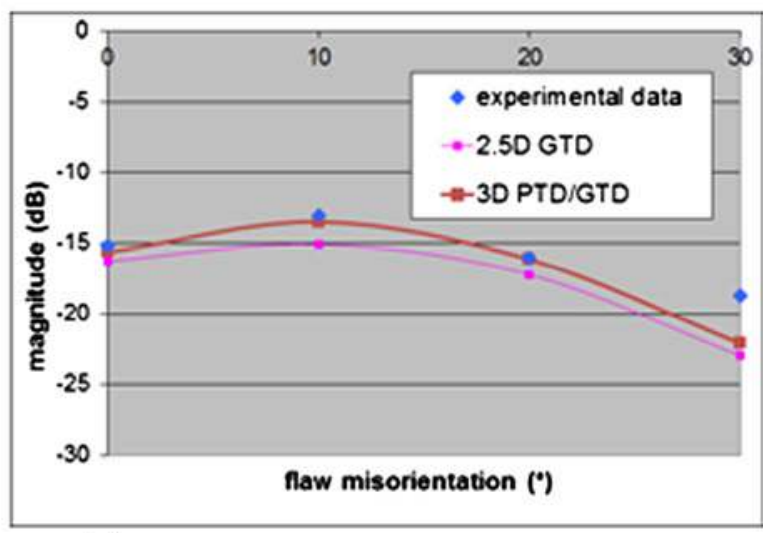

(d)

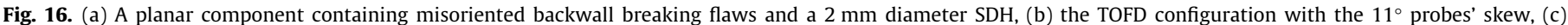
experimental B-scan, (d) validation of the 2.5D GTD and 3D PTD models against the measured echoes from the top tips of misoriented backwall breaking flaws.

and experimental results (GTD results are identical to PTD and are not represented in Fig. 15b).

Let us now turn to our own experimental validation in 3D of the proposed PTD based system model. Several notches have been made in a planar specimen (Fig. 16a). In order to study the influence of both probes' and flaw misorientations, two $6.35 \mathrm{~mm}$ diameter probes emitting $45^{\circ} \mathrm{P}$-waves at $2.25 \mathrm{MHz}$ have been positioned in a TOFD configuration with the $60 \mathrm{~mm}$ Probe Center Separation and misoriented from the $0^{\circ}$ skew angle to the $34^{\circ}$ skew angle. Fig. $16 \mathrm{~b}$ presents a typical case of the $11^{\circ}$ skew. Measurements have been carried out on a rectangular $0^{\circ}$ flaw, three defects (with vertical misorientation of $10-30^{\circ}$ for the top edge) and calibrated against a $2 \mathrm{~mm}$ diameter $\mathrm{SDH}$. The resulting experimental B-Scan is shown in Fig. 16c. Variation of the amplitude of the echo from the top tip with the vertical misorientation is displayed in Fig. 16d. Two models system models are investigated, one based on the so-called 2.5D GTD, which involves the projection of the incoming and scattered wave vectors on the plane normal to the flaw edge and 2D GTD coefficients related to these projections, and the 3D PTD. In the chosen configuration the latter is equivalent to 3D GTD. The agreement between the 3D PTD model and experimental data is good, errors are less than $0.5 \mathrm{~dB}$, except for the $30^{\circ}$ misoriented flaw, for which the signal to noise ratio is low (see Fig. 16c). The 3D PTD based model provides a slight improvement over the 2.5D GTD model, the misorientations being quite small.

The last series of experiments has been performed to evaluate the 3D effect of the flaw skew angle on the edge diffracted echo amplitude. Tests have been carried out on the rectangular backwall breaking flaw studied above using the same pair of transducers as before $\left(2.25 \mathrm{MHz}\right.$ central frequency, $45^{\circ}$ compressional wave, $6.35 \mathrm{~mm}$ diameter). The probes have been positioned in a TOFD configuration with the $60 \mathrm{~mm}$ Probe Center Separation, and the 


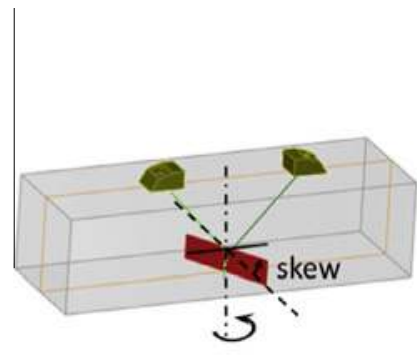

(a)

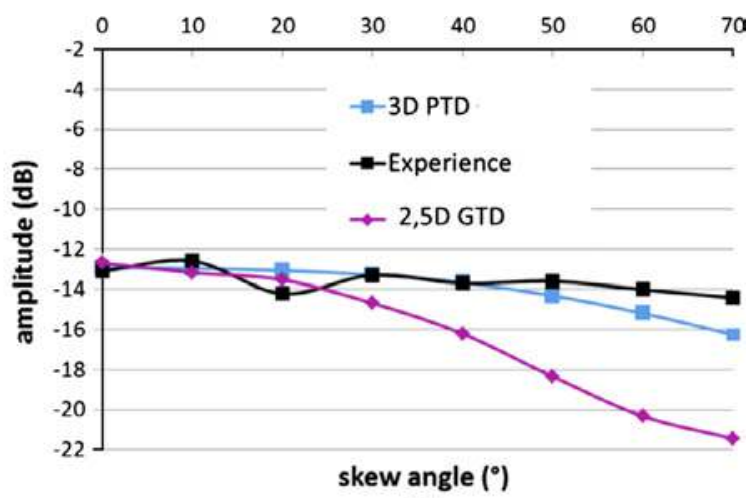

(b)

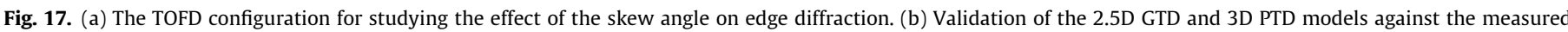
echoes from the top tip of the misoriented backwall breaking flaw.

component has been rotated while the probes remained fixed (Fig. 17a). Such procedure is equivalent to fixing the position of the component and varying the flaw skew angle. In order to observe significant 3D effects the skew angle has been varied from $0^{\circ}$ to $70^{\circ}$.

The experimental and simulated results are presented in Fig. 17b. The experimental results show that the effect of the skew angle on the diffracted echoes is negligible. Moreover, there is a good agreement between experimental and PTD simulated results, with the maximum difference of about $2 \mathrm{~dB}$. Note that when the skew is significant $\left(>30^{\circ}\right)$ the $2.5 \mathrm{D}$ GTD model breaks down.

\section{Conclusions}

An elastodynamic system model based on the Physical Theory of Diffraction (PTD) has been developed to improve simulation of crack echoes. This is a unified model allowing one to simulate both reflection and diffraction phenomena. Numerical comparison with a FEM model has shown a very good overall agreement for P waves and $S$ waves too, provided the latter are calculated at the incidence or observation angles, which are significantly different from critical. Near the critical incidence (resp. observation) angles interference takes place of the reflected (resp. diffracted) $\mathrm{S}$ waves with the head waves. In regions surrounding critical observation angles, the system model based on PTD with smoothing, proposed in this paper, has proved more successful. The PTD model exhibits good performances for crack sizes higher than the wavelength, the overall validity range of PTD $\mathrm{ka}>(\mathrm{ka})_{\max }$ being wider for $\mathrm{P}$ waves $\left((\mathrm{ka})_{\max } \in[1,3]\right.$ about $)$ than for $\mathrm{S}$ waves $\left((\mathrm{ka})_{\max } \in[5,10]\right.$ about $)$. Successful experimental validation of the model has been carried out for diffraction and corner echoes in several typical NDT configurations, 2D and 3D, pulse echo and TOFD.

\section{References}

[1] L.W. Schmerr, S.-J. Song, Ultrasonic Nondestructive Evaluation Systems: Models and Measurements, Springer, 2007.

[2] M. Darmon, S. Chatillon, Main features of a complete ultrasonic measurement model - formal aspects of modeling of both transducers radiation and ultrasonic flaws responses, Open J. Acoust. 3A (2013).

[3] R.K. Chapman, Ultrasonic scattering from smooth flat cracks: an elastodynamic Kirchhoff diffraction theory, CEGB Report, North Western Region NDT Applications Centre, NWR/SSD/82/0059/R, 1982.

[4] J.D. Achenbach, A.K. Gautesen, H. McMaken, Rays methods for waves in elastic solids, Pitman, 1982.

[5] M. Darmon, N. Leymarie, S. Chatillon, S. Mahaut, Modelling of scattering of ultrasounds by flaws for NDT, Ultrasonic Wave Propagation in Non homogeneous Media, vol. 128, Springer, Berlin, 2009, pp. 61-71.

[6] M. Spies, Kirchhoff evaluation of scattered elastic wavefields in anisotropic media, J. Acoust. Soc. Am. 107 (5) (2000) 2755-2759.
[7] V. Dorval, S. Chatillon, B. Lu, M. Darmon, S. Mahaut, A general Kirchhoff approximation for echo simulation in ultrasonic NDT, in: 38th Annual Review of Progress in Quantitative Nondestructive Evaluation (QNDE), 2012, vol. 1430, pp. 193-200.

[8] G. Baskaran, K. Balasubramaniam, C.V. Krishnamurthy, C.L. Rao, Ray based model for the ultrasonic time-of-flight diffraction simulation of thin walled structure inspection, J. Press. Vessel Technol. 127 (3) (2005) 262-268.

[9] S.K. Nath, Effect of variation in signal amplitude and transit time on reliability analysis of ultrasonic time of flight diffraction characterization of vertical and inclined cracks, Ultrasonics 54 (3) (2014) 938-952.

[10] M. Darmon, S. Chatillon, S. Mahaut, L. Fradkin, A. Gautesen, Simulation of disoriented flaws in a TOFD technique configuration using GTD approach, in: 34th Annual Review of Progress in Quantitative Nondestructive Evaluation, vol. 975, 2008, pp. 155-162.

[11] S. Kolkoori, K. Chitti Venkata, K. Balasubramaniam, Quantitative simulation of ultrasonic time of flight diffraction technique in 2D geometries using Huygens-Fresnel diffraction model: theory and experimental comparison, Ultrasonics 55 (2015) 33-41.

[12] G.F. Miller, H. Pursey, The field and radiation impedance of mechanical radiators on the free surface of a semi-infinite isotropic solid, Proc. R. Soc London Math. Phys. Eng. Sci. 223 (1155) (May 1954) 521-541.

[13] A. Lhemery, Modèles théoriques et études pratiques en imagerie ultrasonore large bande; applications au contrôle non destructif des matériaux, Habilitation à diriger des recherches, Université Paris VII Denis Diderot-2000.

[14] D. Gridin, The radiating near field of a circular normal transducer of arbitrary apodization on an elastic half-space, J. Acoust. Soc. Am. 106 (3) (1999) 12371246.

[15] D. Gridin, L.J. Fradkin, The high-frequency asymptotic description of pulses radiated by a circular normal transducer into an elastic half-space, J. Acoust. Soc. Am. 104 (6) (1998) 3190-3198.

[16] P.Y. Ufimtsev, Fundamentals of the Physical Theory of Diffraction, John Wiley \& Sons, 2007.

[17] V. Zernov, L. Fradkin, M. Darmon, A refinement of the Kirchhoff approximation to the scattered elastic fields, Ultrasonics 52 (7) (2012) 830-835.

[18] Civa software website, <http://www.civa.cea.fr> and <http://www. extende.com/>.

[19] S. Mahaut, M. Darmon, S. Chatillon, F. Jenson, P. Calmon, Recent advances and current trends of ultrasonic modelling in CIVA, Insight 51 (2) (2009) 78-81.

[20] P. Calmon, S. Mahaut, S. Chatillon, R. Raillon, CIVA: An expertise platform for simulation and processing NDT data, Ultrasonics 44 (Supplement) (2006) e975-e979.

[21] N. Gengembre, A. Lhémery, Pencil method in elastodynamics: application to ultrasonic field computation, Ultrasonics 38 (1-8) (2000) 495-499.

[22] N. Gengembre, A. Lhémery, Calculation of wideband ultrasonic fields radiated by water-coupled transducers into heterogeneous media, in: D.O. Thompson, D.E. Chimenti (Eds.), Review of Progress in Quantitative Nondestructive Evaluation, Springer, US, 1999, pp. 1131-1138.

[23] M. Darmon, P. Calmon, B. Bele, An integrated model to simulate the scattering of ultrasounds by inclusions in steels, Ultrasonics 42 (1) (2004) 237-241.

[24] N. Leymarie, P. Calmon, T. Fouquet, A. Schumm, A semi-analytical FEM hybrid model for ultrasonic simulation of complicated wave defect interaction, in: International Conference on NDE in relation to structural Integrity for Nuclear and Pressurised Component 2006, 2006.

[25] A. Kamta Djakou, M. Darmon, C. Potel, Elastodynamic models for extending the Geometrical Theory of Diffraction to finite length defect, Submitt. IEEE Trans. Ultrason. Ferroelectr. Freq. Control.

[26] L.J. Fradkin, M. Darmon, S. Chatillon, P. Calmon, The triple singularity: a seminumerical model for the near-critical angle scattering, Submitt. J. Acoust. Soc Am.

[27] L. Fradkin, M. Harmer, M. Darmon, Edge diffraction coefficients around critical rays, J. Phys.: Conf. Ser. 498 (2014) 012010. 
[28] E. Becache, P. Joly, C. Tsogka, Application of the fictitious domain method to 2D linear elastodynamic problems, J. Comput. Acoust. 9 (2001) 1175-1202.

[29] E. Becache, P. Joly, C. Tsogka, An analysis of new mixed finite elements for the approximation of wave propagation problems, SIAM J. Numer. Anal. 37 (2000) 1053-1084.

[30] F.A. Ravenscroft, K. Newton, C.B. Scruby, Diffraction of ultrasound by cracks: comparison of experiment with theory, Ultrasonics 29 (1) (1991) 29-37.

[31] R. Raillon, G. Toullelan, M. Darmon, S. Lonne, Experimental study for the validation of CIVA predictions in TOFD inspections, in: 10th International
Conference on NDE in Relation to Structural Integrity for Nuclear and Pressurized Components, Cannes, October 2013, 2013.

[32] P.S. Keogh, High-frequency scattering by a griffith crack II: incident plane and cylindrical waves, Q. J. Mech. Appl. Math. 38 (2) (May 1985) 205-232.

[33] R. Raillon-Picot, G. Toullelan, M. Darmon, P. Calmon, S. Lonné, Validation of CIVA ultrasonic simulation in canonical configurations, in: World Conference of Non Destructive Testing (WCNDT) 2012, 2012.

[34] M. Darmon, A. Ferrand, V. Dorval, S. Chatillon, S. Lonné, Recent modelling advances for ultrasonic TOFD inspections, AIP Conf. Proc. 1650 (2015) 17571765. 